\title{
Bleeding Complications in Patients Receiving Direct Oral Anticoagulant Therapy in the Post Clinical Trial General Practice
}

\author{
Subhash Edupuganti, Catherine Xie, Mary Wyrzykowski, Elizabeth Wey, Ming Xie* \\ Department of Pathology, Oakland University School of Medicine, William Beaumont Hospital, Troy, Michigan, USA
}

Email address:

ming.xie@beaumont.org (Ming Xie)

${ }^{*}$ Corresponding author

\section{To cite this article:}

Subhash Edupuganti, Catherine Xie, Mary Wyrzykowski, Elizabeth Wey, Ming Xie. Bleeding Complications in Patients Receiving Direct Oral Anticoagulant Therapy in the Post Clinical Trial General Practice. American Journal of Clinical and Experimental Medicine.

Vol. 5, No. 3, 2017, pp. 64-68. doi: 10.11648/j.ajcem.20170503.12

Received: January 31, 2017; Accepted: February 14, 2017; Published: April 10, 2017

\begin{abstract}
Dabigatran, apixaban and rivaroxaban are direct oral anticoagulants (DOACs) recently approved for patients with venous thromboembolism. Therapy-induced hemorrhage remains a major complication in these patients. This study retrospectively reviews the hemorrhagic complications associated with DOACs in the general practice and the related clinical implications. The electronic medical charts of 2255 patients with prolonged PTT tests during August 2015 to April 2016 at William Beaumont Health System - Troy were retrospectively reviewed. Patients with prolonged PTT and simultaneously receiving DOAC's were identified. Hemorrhagic complications associated with DOAC therapy and the related clinical information were analyzed. $517(22.9 \%)$ patients were identified receiving DOAC therapy. Among these, DOAC therapyassociated hemorrhages were recorded in 85 patients (16.4\%). Apixaban had a significantly lower incidence of hemorrhage $(8.8 \%)$ than rivaroxaban $(21.0 \%)$ and dabigatran $(27.9 \%)$. The most common hemorrhage was GI bleeding (7.0\% overall); its incidence was significantly higher in dabigatran $(18.6 \%)$ than apixaban $(4.1 \%)$ and rivaroxaban $(7.4 \%)$; but no significant difference between apixaban and rivaroxaban ( $p>0.05$ ). GI bleeding produced anemia in 13 patients who received additional treatments with occasional blood transfusions. Since GI bleeding is the most common bleeding complication and may cause anemia in patients receiving DOAC therapy, routine studies for GI bleeding should be encouraged to reduce the complications of GI bleeding. Apixaban has significantly lower incidence of bleeding complications, it may be a better choice in patients with increased bleeding risk overall. However, apixaban may not improve the bleeding risk in patients with GI bleeding associated with rivaroxaban.
\end{abstract}

Keywords: Anticoagulants, Gastrointestinal Hemorrhage, Activated Partial Thromboplastin Time, Apixaban, Rivaroxaban, Dabigatran

\section{Introduction}

As direct oral anticoagulants (DOACs), apixaban, dabigatran and rivaroxaban have been approved for the prevention and treatment of thromboembolic diseases, and have gained wide use recently to replace the traditional oral vitamin $\mathrm{K}$ antagonists (Warfarin) therapy $[1,2,3]$. Dabigatran is a direct thrombin inhibitor, while apixaban and rivaroxaban are both direct factor $\mathrm{Xa}$ inhibitors $[4,5]$. DOACs are reported to display predictable pharmacokinetic profiles, with fewer drug-drug interactions and shorter half- lives. They are administered with fixed dosing without the need for routine laboratory monitoring [6, 7]. DOAC therapies have demonstrated similar to superior efficacy in reducing the risk of thromboembolism when compared with warfarin therapy $[8,9,10]$.

While the direct oral anticoagulants do demonstrate lower risk for intracranial hemorrhage, both major and non-major bleeding events have been reported in patients receiving these medications [11, 12], and their management remains a cause of major concern [13]. Until now, most of the data on DOAC therapy is derived from clinical trials with limited 
postmarketing evaluations, and the focus has been on the comparison of bleeding risk between DOAC therapy and warfarin $[14,15]$. The differences between the various DOAC agents have not been studied extensively and need further clinical investigation. Partial thromboplastin time (PTT) becomes prolonged in most patients receiving DOAC therapy [16] and is a sensitive laboratory test to reflect the anticoagulation effects by DOAC agents [17]. PTT is also the most commonly used laboratory test to evaluate the hemorrhagic risk in general practice. This study is designed to retrospectively review the bleeding complications associated with different DOAC agents in patients with prolonged PTT and the clinical outcomes in these patients.

\section{Materials and Methods}

Patient population:

From August 2015 to April 2016, 2255 patients with prolonged PTT test results were identified at the coagulation laboratory, William Beaumont hospital, Troy, Michigan. Among them, 517 patients were identified with prolonged PTT results while on DOAC therapy at the same time. The electronic medical charts from these patients were retrospectively reviewed and analyzed for hemorrhagic complications and subsequent clinical outcomes.

DOAC therapy information:

The clinical indications for DOAC therapy are atrial fibrillation (AF), deep vein thrombosis (DVT) and pulmonary embolism (PE). Each patient was given one of the followings dosages: apixaban was given $2.5 \mathrm{mg}$ or $5.0 \mathrm{mg}$ bid; dabigatran $75 \mathrm{mg}$ or $150 \mathrm{mg}$ bid; or rivaroxaban $15 \mathrm{mg}$ or $20 \mathrm{mg}$ bid. No patient received 2 or 3 DOAC medications at the same time. All patients received DOAC therapy for more than a month. Other antithrombotic therapy such as antiplatelet agents (aspirin and clopidgrel), heparin, low molecular weight heparin (lovenox) and warfarin was also recorded.

Hemorrhagic complications associated with DOAC therapy:

Bleeding complications were recorded as documented in the medical charts. Some patients had bleeding with multiple organ system involvement, in which case, we recorded the total number of patients for overall hemorrhagic incidence associated with DOAC therapy and the number of hemorrhages at different anatomic locations for subclassification of these hemorrhagic complications, such as GI bleeding, hematuria, bruises, etc. Analysis of hemorrhagic complications includes the followings:

Overall incidence of hemorrhagic complications: The overall incidence of bleeding associated with DOAC therapy was reviewed.

Anatomic distribution of hemorrhagic complications: Hemorrhage was sub-classified based on the anatomic location or organ system involved with further attention to the possible underlying medical conditions.

Comparison of hemorrhages between different DOAC agents: The different anatomic locations and organ systems involved with hemorrhage were compared between different DOAC agents. Special attention was paid to GI bleeding, as it was the single most common bleeding complication in these patients.

Clinical outcomes and management:

Hemorrhage induced anemia and other related complications or mortalities were reviewed. Clinical management of patients with DOAC therapy associated bleeding was recorded, including the followings: discontinuing DOAC therapy, switching to a different antithrombotic agent, or temporarily holding DOAC therapy and resuming the same medication after the patient clinical condition stabilized. Transfusion of blood products and other supportive treatments for bleeding complications were also reviewed.

Statistical analysis:

Student's T test was used for the calculation of $\mathrm{P}$ value to determine the significance of the difference between 2 patient groups.

\section{Results}

\section{General patient information:}

There were 517 patients with prolonged PTT while receiving DOAC therapy, consisting of $22.9 \%$ (517/2255) of the total number of patients with prolonged PTT at the same time. The 517 patients included 273 males and 244 females, with the median age of 73 , ranging from 22 to 90 . There were $217(42.0 \%)$ patients receiving apixaban; 43 (8.3\%) patients receiving dabigatran; and $257(49.7 \%)$ patients receiving rivaroxaban.

Hemorrhagic complications with DOAC therapy:

Among the 517 patients with prolonged PTT, there were $85(16.4 \%)$ patients who experienced hemorrhagic complications as recorded in their medical charts. As shown in table 1 , the hemorrhagic incidence was $8.8 \%(19 / 217)$ in patients with apixaban; 27.9\% (12/43) in patients with dabigatran; and $21.0 \%(54 / 257)$ in patients with rivaroxaban. The difference was statistically significant between apixaban and dabigatran $(\mathrm{p}<0.01)$, and between apixaban and rivaroxaban $(\mathrm{p}<0.01)$. There was no statistically significant difference between dabigatran and rivaroxaban $(p>0.05)$.

Table 1. Patients with hemorrhagic complications related to DOAC therapy.

\begin{tabular}{lllll}
\hline DOACs & \# with Hemorrhage & Total \# & Percentage & p value \\
\hline Apixaban (A) & 19 & 217 & $8.8 \%$ & $<0.01$ (A and B) \\
Dabigatran (B) & 12 & 43 & $27.9 \%$ & $<0.01$ (A and C). \\
Rivaroxaban (C) & 54 & 257 & $21.0 \%$ & \\
\hline
\end{tabular}


Anatomic location of the hemorrhagic complications:

Overall, GI bleeding was the most commonly recorded hemorrhagic complication in these patients (7.0\%). The anatomic distributions of hemorrhagic complications are shown in Figure 1 as follows: GI bleeding (39.1\%), hematuria $(16.3 \%)$, epistaxis $(16.3 \%)$, bruises $(12.0 \%)$, hematoma $(7.6 \%)$ and others $(8.7 \%)$.

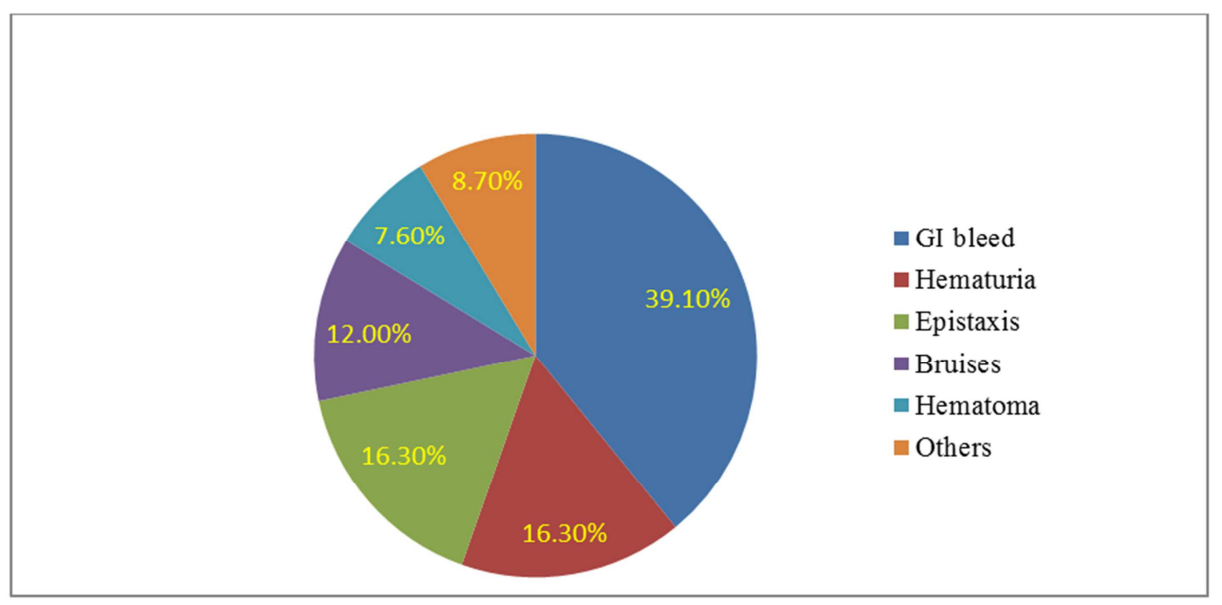

Figure 1. Frequency of hemorrhagic complications in patients receiving DOAC therapy.

Comparison between apixaban with rivaroxaban:

Although the overall incidence of bleeding complications was statistically higher in patients receiving rivaroxaban than apixaban, there was no significant difference in the incidence of GI bleeding between these two medications ( $p>0.05$, Table 2). In fact, among the patients with hemorrhage and prolonged PTT, the percentage of GI bleeding in patients with apixaban was slightly higher than that with rivaroxaban
(40.9\% vs. $33.9 \%)$. As shown in Figure 2, there were some differences in anatomic location of their bleeding complications between apixaban and rivaroxaban, and the incidence of non-GI bleeding was relatively higher in patients receiving rivaroxaban than apixaban, especially epistaxis (19.6\% vs. $13.6 \%)$ and bruises $(16.0 \%$ vs. $4.5 \%)$. Only 1 patient was reported to have intracranial hemorrhage after minor injury while on rivaroxaban.

Table 2. Patients with GI bleed related to DOAC therapy.

\begin{tabular}{|c|c|c|c|c|}
\hline Groups & \# with GI bleed & Total \# & Percentage & p value \\
\hline Apixaban (A) & 9 & 217 & $4.1 \%$ & \\
\hline Dabigatran (B) & 8 & 43 & $18.6 \%$ & $<0.01(\mathrm{~A}$ and $\mathrm{B})$ \\
\hline Rivaroxaban (C) & 19 & 257 & $7.4 \%$ & $>0.05(\mathrm{~A}$ and $\mathrm{C})$ \\
\hline
\end{tabular}

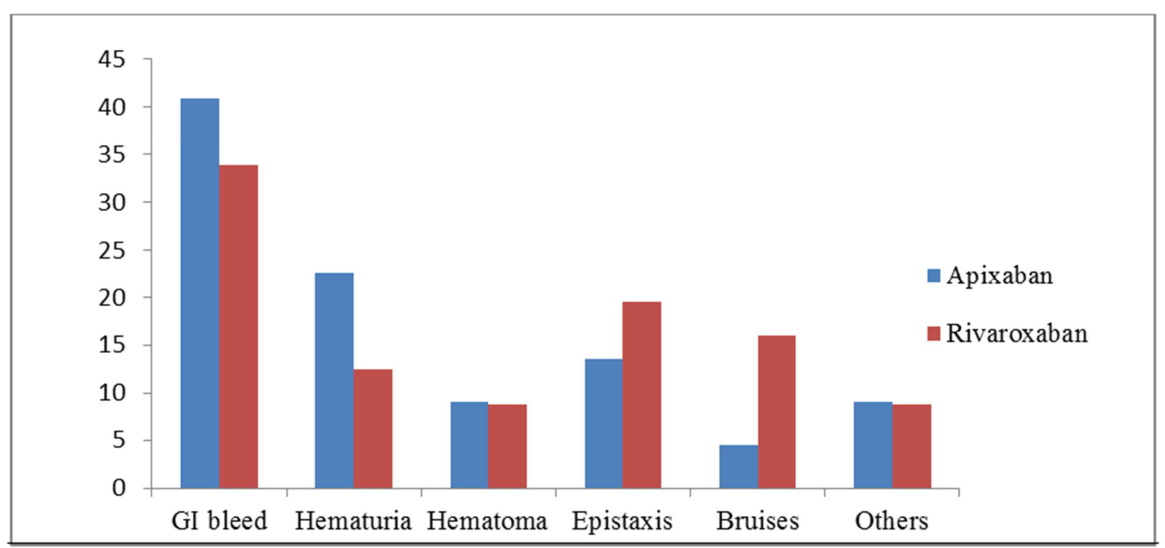

Figure 2. Anatomic locations of hemorrhage between Apixaban with Rivaroxaban (\%).

Clinical management of the DOAC therapy related hemorrhage:

Among the 85 patients with hemorrhage, there were 13 patients reported with hemorrhage associated anemia that is $15.3 \%(13 / 85)$ of the patients with bleeding complications and $2.5 \%(13 / 517)$ of the total patients receiving DOAC therapy with prolonged PTT. All of these 13 patients had GI bleeding, and one of them had hematuria as well. Two patients had red blood cell transfusions secondary to the anemia. There were no reported mortalities directly related to the hemorrhagic complications due to DOAC therapy.

DOAC therapy was discontinued or temporarily held in all 
of the patients with hemorrhagic complications. After the clinical condition was stabilized, some patients resumed the same DOAC therapy and some switched to a different class of anticoagulants, including warfarin, clopidgrel or lovenox, as needed according to the patient clinical conditions. A few patients were switched from rivaroxaban to apixaban. No patient was switched from apixaban to rivaroxaban or dabigatran.

\section{Discussion}

Bleeding complications remain the major concern for patients receiving DOAC therapy, especially when no specific antidotes are available for most of the DOAC therapies $[18,19,20]$. In this study including 517 patients on DOAC therapy with prolonged PTT in the postmarketing general practice, 85 patients were recorded with hemorrhagic complications (16.4\%). When compared among the different DOACs, patients receiving apixaban had a lower hemorrhagic rate $(8.8 \%)$ than patients with dabigatran and rivaroxaban $(27.9 \%$ and $21.0 \%)$, with statistical significance $(\mathrm{p}<0.01)$.

Overall, GI bleeding (7.0\%) was the most commonly recorded hemorrhage in these patients, which is similar to previous reports $[15,21]$. Hematuria and epistaxis were the next most common hemorrhagic complication after GI bleeding. Dabigatran had the highest incidence of GI bleeding $(18.6 \%)$, followed by rivaroxaban $(7.4 \%)$ and apixaban $(4.1 \%)$.

Both apixaban and rivaroxaban are direct factor $\mathrm{Xa}$ inhibitors. However, the anatomic distribution of hemorrhagic complications appears different between these 2 agents in this study. The overall hemorrhagic incidence was statistically higher in patients receiving rivaroxaban than apixaban $(\mathrm{p}<0.01)$. There was no significant difference in the incidence of GI bleeding $(\mathrm{P}>0.05)$ between these 2 agents. This is likely because the incidence of non-GI bleeding is higher in patients on rivaroxaban, particularly epistaxis and bruises, which rarely cause clinically significantly anemia.

In addition to being the most common hemorrhagic complication in this study, GI bleeding was also the hemorrhagic complication causing clinically significant anemia in 13 patients, with occasional transfusion of blood products. There was only one patient reported to have intracranial hemorrhage while on rivaroxaban after minor injury. There were no reported mortalities associated with the hemorrhagic complications identified in this study.

Discontinuation of DOAC therapy was recorded in the patients with hemorrhagic complication and prolonged PTT. After further clinical assessment and stabilization of patient clinical condition, some patients resumed the same DOAC therapy and some switched to a different class of anticoagulants, including warfarin, clopidgrel or lovenox. A few patients were switched from rivaroxaban to apixaban after the bleeding episodes. But no patients were switched from apixaban to rivaroxaban or dabigatran.

Post marketing studies on DOAC therapy have yielded some data similar to the clinical trial results $[22,23]$. The hemorrhagic incidence reported in this study in patients with prolonged PTT during DOAC therapy may not reflect the hemorrhagic incidence in all patients with DOAC therapy. However, prolonged PTT may indicate significant coagulopathy, which may be therapeutic, but also a risk factor for hemorrhage. By selecting patients with prolonged PTT, this study is focused more on patients with increased hemorrhagic risk and may provide more information in identifying these patients in the general practice.

\section{Conclusion}

The overall risk of hemorrhage is lower in patients receiving apixaban, compared to dabigatran and rivaroxaban. However, the anatomic distribution of bleeding complication seems slightly varied between apixaban and rivaroxaban, with no significant difference in the incidence of GI bleeding between these 2 agents in this study. GI bleeding is the most common hemorrhage and also the most important complication responsible for clinically significant anemia with significant impact on patient management. It may be reasonable to perform regular screening studies for GI bleeding in patients receiving DOAC therapy with prolonged PTT. Apixaban may be an alternative for patients with non-GI bleeding while receiving rivaroxaban. This practice may be questionable in patients with GI bleeding. Additional clinical studies with a larger patient sample are needed to further evaluate the safety of DOAC therapy in general practice.

\section{References}

[1] Bacchus F and Schulman S. Clinical experience with the new oral anticoagulants for treatment of venous thromboembolism. Arterioscler Thromb Vasc Biol. March 2015, P1-7.

[2] Yao X, Abraham NS, Sangaralingham LR, Bellolio MF, McBane RD, Shah ND and Noseworthy PA. Effectiveness and safety of dabigatran, rivaroxaban, and apixaban versus warfarin in nonvalvular atrial fibrillation. J Am Heart Assoc. 2016;.116.003725.

[3] Mantha $\mathrm{S}$ and Ansell J. Indirect comparison of dabigatran, rivaroxaban, apixaban and edoxaban for the treatment of acute venous thromboembolism. J Thromb Thrombolysis. 2015; 39 (2): $155-65$

[4] Riva N and Ageno W. Pros and cons of vitamin K antagonists and non-vitamin $\mathrm{K}$ antagonist oral anticoagulants. Semin Thromb Hemost 2015; 41 (2): 178-187.

[5] Ebright $\mathbf{J}$ and Mousa SA. Oral anticoagulants and status of antidotes for the reversal of bleeding risk. Clinical and Applied Thrombosis/Hemostasis 2015; 21 (2): 105-114.

[6] Gehrie E and Tormey C. Novel oral anticoagulants: efficacy, laboratory measurement, and approaches to emergent reversal. Arch Pathol Lab Med 2015; 139 (5): 687-692.

[7] Pollack CV. Introduction to direct oral anticoagulants and rationale for specific reversal agents. Am J Med. 2016; 129: S31-S32. 
[8] Ufer M. Comparative efficacy and safety of the novel oral anticoagulants dabigatran, rivaroxaban, and apixaban in preclinical and clinical development. Thromb Haemost 2010; 103 (3): 572.

[9] Ruff CT, Giugliano RP, Braunwald E, et al. Comparison of the efficacy and safety of new oral anticoagulants with warfarin in patients with atrial fibrillation: a meta-analysis of randomized trials. Lancet 2014; 383: 955-62.

[10] Alotaibi G, Alsaleh K, Wu C, and Mcmurty MS. Dabigatran, rivaroxaban and apixaban for the extended venous thromboembolism treatment: network meta-analysis. Int Angiol, 2014; 33 (4): 301-8.

[11] Abraham NS, Singh S, Alexander GC, Heien H, Haas LR, Crown W and Shah ND. Comparative risk of gastrointestinal bleeding with dabigatran, rivaroxaban, and warfarin: population based cohort study. BMJ 2015; 350: h1857.

[12] Tran H, Joseph J, Young L, McRae S, Curnow J, Nandurkar $\mathrm{H}$, Wood P, McLintock C. New oral anticoagulants: a practical guide on prescription, laboratory testing and periprocedural/bleeding management. Australasian Society of Thrombosis and Haemostasis. Intern Med J. 2014; 44 (6): 525-36.

[13] Franchini M, Bonfanti C and Mannucci PM. Management of bleeding associated with new oral anticoagulants. Semin Thromb Hemost 2015; 41 (7): 788-801.

[14] Villines TC and Peacock WF. Safety of direct oral anticoagulants: Insights from postmarketing studies. Am J Med. 2016; 129: S41-S46.

[15] Eikelboom J and Merli G. Bleeding with direct oral anticoagulants vs warfarin: Clinical experience. Am J Med. 2016; 129: S33-S40.

[16] Favaloro EJ, Lippi G, Koutts J. Laboratory testing of anticoagulants: the present and the future. Pathology 2011; 43 (7): 682-692.
[17] Mary Wyrzykowski, Victoria Walley-Goike and Ming Xie. Retrospective analysis of abnormal partial thromboplastin time results in patients receiving new oral anticoagulants. Am J Clin Pathol 2016; 146 (suppl 1): 106 (abstract).

[18] Halperin JL, Hankey GJ, Wojdyla DM, et al. Efficacy and safety of rivaroxaban compared with warfarin among elderly patients with nonvalvular atrial fibrillation in the rivaroxaban once daily, oral, direct factor $\mathrm{Xa}$ inhibition compared with vitamin $\mathrm{K}$ antagonism for prevention of stroke and embolism trial in atrial fibrillation (ROCKET AF). Circulation 2014; 130: $138-46$.

[19] McMahon BJ and Kwaan HC. The new or non-vitamin K antagonist oral anticoagulants: what have we learned since their debut. Semin Thromb Hemost 2015; 41 (2): 188-194.

[20] Forman DE and Goyette RE. Oral anticoagulation therapy for elderly patients with atrial fibrillation: utility of bleeding risk covariates to better understand and moderate risks. Clinical and Applied Thrombosis/Hemostasis 2014; 20 (1): 5-15.

[21] Holster IL, Valkhoff VE, Kuipers EJ, et al. New oral anticoagulants increase risk for gastrointestinal bleeding: a systematic review and meta-analysis. Gastroenterology 2013; 145: 105-12.

[22] Villines TC, Schnee J, Fraeman K, et al. The comparative safety and effectiveness of the oral anticoagulant (OAC) dabigatran versus warfarin utilized in a large healthcare system in non-valvular atrial fibrillation (NVAF) patients. Circulation. 2014; 130 (suppl 2): A18353.

[23] Tepper P, Mardekian J, Masseria C, et al. Real-world comparison of bleeding risks among non-valvular atrial fibrillation patients on apixaban, dabigatran, rivaroxaban: cohorts comprising new initiators and/or switchers from warfarin. Eur Heart J. 2015; 36 (suppl): 339. 